PoS $\quad \begin{aligned} & \text { PROCEEDINGS } \\ & \text { OF SCIENCE }\end{aligned}$

\title{
Heavy Flavour production results with ATLAS at
} $\sqrt{s}=7,8$ and $13 \mathrm{TeV}$

\section{Canale on behalf of the ATLAS Collaboration*}

Università di Napoli "Federico II", Dipartimento di Fisica "Ettore Pancini”, Napoli and I.N.F.N. Napoli

E-mail: Vincenzo.Canale@cern.ch

We present some recent results on heavy flavour production obtained with ATLAS during the LHC runs in 2011-2012 at $\sqrt{s}=7,8 \mathrm{TeV}$ and in 2015 at $\sqrt{s}=13 \mathrm{TeV}$.

Fourth Annual Large Hadron Collider Physics

13-18 June 2016

Lund, Sweden

${ }^{*}$ Speaker. 


\section{Introduction}

The production of heavy flavours (HF) at hadron colliders provides particular opportunity to study the theory of Quantum Chromodynamics (QCD), in particular the boundary of the perturbative and non-perturbative regimes. At the LHC, due to the high energy available $\sqrt{s}=7,8$, and $13 \mathrm{TeV}$, it is possible to explore new kinematic regions (e.g., $\operatorname{high}^{1} p_{T}$ up to $\sim 10^{2} \mathrm{GeV}$ ) to test the predictions of various theoretical models for both quarkonium and open state production. In most cases, corrections over the leading order (LO) are available (e.g., next-to-leading (NLO), next-to-leading-log (NLL)) and LHC data may allow accurate quantitative comparisons of data with QCD predictions to discriminate among various HF production models both in the quarkonium sector (color singlet model (CSM) [1], non relativistic QCCD model (NRQCD) [2]) and in the open state one (FONLL model [3], GM-VFNS scheme [4], NLO-MC matched calculations [5]).

\section{Experimental aspects}

\subsection{Data sample and detector performances}

ATLAS is a multipurpose apparatus to study $p p$ interactions at LHC, the detailed description and performances of the detector can be found in [6]. The data sample corresponds to the LHC integrated luminosities of: (a) $5.1 \mathrm{fb}^{-1}$ at $\sqrt{s}=7 \mathrm{TeV}$ in 2011; (b) $21.3 \mathrm{fb}^{-1}$ at $\sqrt{s}=8 \mathrm{TeV}$ in 2012; (c) $3.9 \mathrm{fb}^{-1}$ at $\sqrt{s}=13 \mathrm{TeV}$ in 2015 . Regarding the analysis on HF, the main detector components are: (a) the Muon System (MS) with tracking capability up to pseudorapidity $|\eta| \leq 2.7$, with momentum resolution $\sigma_{p_{T}} / p_{T}=0.06$ up to $60 \mathrm{GeV}$; (b) the Inner Detector (ID) which reconstructs tracks up to $|\eta| \leq 2.5$ with efficiency $\varepsilon_{t r k}=0.99$. The vertexing accuracy (primary and secondary) results in a resolution on the track impact parameter ${ }^{2} \sigma_{\delta} \simeq 10 \mu \mathrm{m}$. The trigger for HF physics is based on di-muons up to $|\eta| \leq 2.4$ and with several combinations of transverse momentum thresholds $p_{T}^{(\mu)}=4$ and $6 \mathrm{GeV}$.

The invariant mass distribution of a specific decay channel $Q \rightarrow$ final is the key-tool for HF analyses; the typical resolutions are in the range $(50 \div 100) \mathrm{MeV}$ for $\mu \mu$ pairs or other exclusive states, and the observed signal/background ratios are high. From vertices reconstruction, it is possible to obtain the pseudo proper lifetime of the reconstructed state, $t=L_{T} \cdot \frac{m_{Q}}{P_{T}}$ where $L_{T}$ is the transverse decay length projected along the $\vec{P}_{T}$ of the decaying particle, which allows to reduce background or disentangle prompt and non-prompt ${ }^{3}$ components of the signal.

\subsection{Cross section evaluation}

Experimentally, for each channel $Q \rightarrow$ final, the double differential cross section in $p_{T}$ and rapidity $y$, is obtained from

$$
\frac{d^{2} \sigma_{Q}}{d p_{T} d y} \cdot B R(Q \rightarrow \text { final })=\frac{N_{\text {corr }}^{Q \rightarrow \text { final }}}{\mathscr{L} \cdot \Delta p_{T} \cdot \Delta y}
$$

where $\mathscr{L}$ is the integrated luminosity which may vary for each analysis (trigger, etc...), $\Delta p_{T}$ and $\Delta y$ are the bin sizes of the differential variables within the typical ranges $O(10) \mathrm{GeV} \leq p_{T} \leq$

\footnotetext{
${ }^{1} p_{T}$ is the transverse momentum to the direction of colliding beams.

${ }^{2} \delta$ is the distance of closest approach to the primary vertex.

${ }^{3}$ Non-prompt component originates from decays of b-hadrons.
} 
$O(100) \mathrm{GeV}$ and $0 \leq|y| \leq 2$, and $N_{\text {corr }}^{Q \rightarrow \text { final }}$ is the signal yield corrected for efficiency, acceptance and background subtraction.

The extraction of the yield is obtained with unbinned maximum likelihood methods. The signal is parametrized with combinations of Gaussian and Crystall Ball shapes for the mass distributions and negative exponential for the pseudo proper time, which are convoluted with the experimental resolution whose parameters are obtained from the fit or derived preliminary from data. The background is described in different ways (e.g Chebyshev polynomial, exponential, ec...). The systematic uncertainties corresponding to the fitting procedure are in general at the level of a few $\%$.

The signal yield is corrected with weights which take into account efficiency for tracking, reconstruction, and acceptance: $w^{-1}=\varepsilon^{(\text {reco. })} \cdot \varepsilon^{(\text {trigger })} \cdot \mathscr{A}$. Reconstruction and trigger efficiencies are obtained with data driven methods (e.g., tag and probe for the trigger) in order to reduce the systematic uncertainties which amount typically to $(5 \div 10) \%$. A typical average trigger weight bidimensional map, as function of $p_{T}$ and $y$ of a $\mu \mu$ pair, is shown in figure 1(a). The acceptance corrections, to recover the visible phase space in $p_{T}$ and $y$ for comparison with QCD predictions, are obtained with simulation. They depend on the unknown polarization status of Q (spin alignment [7]). The correction is evaluated for the isotropic case and an envelope of variations is computed for the various polarizations. For example, figure 1(b) shows this acceptance for the $J / \psi$ as a function of the $p_{T}^{J / \psi}$ for the different polarizations. The high $p_{T}$ regions are more interesting because the variation envelope is reduced at $\sim 15 \%$ level.

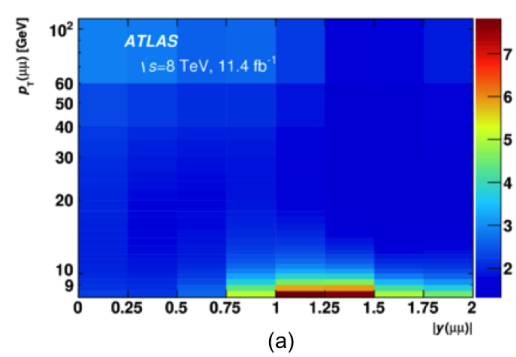

(a)

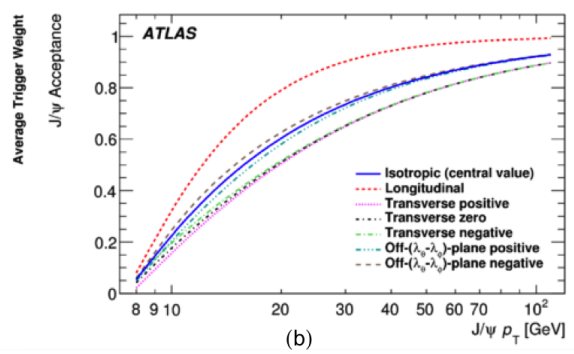

(b)

Figure 1: Correction factors for cross section evaluation [8]: (a) $p_{T}-y$ trigger weight map; (b) acceptance correction vs $p_{T}$ for different production polarization status.

\section{Charmonium production}

First studies on prompt charmonium production were based on the measurement of $J / \psi$. However, the comparisons with theoretical models are difficult for $J / \psi$, due to the uncertain contributions from higher $(c \bar{c})$ states which decays to $J / \psi$ (feed-down effect). To overtake feed-down uncertainties, analyses were performed to include the study of the $\psi(2 s)$, the S-wave $c \bar{c}$ state just below the open charm threshold for which the feed down effect is reduced.

\section{1 $\mathrm{J} / \psi$ and $\psi_{2 s}$ production at $\sqrt{s}=7 \mathrm{TeV}$ and $\sqrt{s}=8 \mathrm{TeV}$}

ATLAS has studied the production of $J / \psi \rightarrow \mu^{+} \mu^{-}$and $\psi(2 s) \rightarrow \mu^{+} \mu^{-}$for data at $\sqrt{s}=7 \mathrm{TeV}$ $\left(\mathscr{L}=2.1 \mathrm{fb}^{-1}\right)$ and $\sqrt{s}=8 \mathrm{TeV}\left(\mathscr{L}=11.4 \mathrm{fb}^{-1}\right)[8]$. The yields are extracted from a fit to the com- 
bined mass-time distribution in the different $p_{T}-y$ intervals. An example of the fit projections are shown in figure 2 in which the various $J / \psi, \psi(2 s)$, prompt an non-prompt contributions are clearly visible. The differential cross sections for prompt production, as a function of $p_{T}$ for different $y$ intervals, are shown in figure 3: (a) for $J / \psi$ at $\sqrt{s}=7 \mathrm{TeV}$, (b) for $\psi(2 s)$ at $\sqrt{s}=8 \mathrm{TeV}$. The NRQCD predictions are superimposed and the ratio theory/data is reported on a separate plots. A fair agreement, between the theoretical calculations and the data points, is observed for the whole $p_{T}$ range; and the ratio of theory to data does not depend on rapidity. Similarly the non-prompt differential cross sections are are shown in figure 4: (a) for $J / \psi$ at $\sqrt{s}=8 \mathrm{TeV}$, (b) for $\psi(2 s)$ at $\sqrt{s}=7 \mathrm{TeV}$. The comparison is performed with FONLL predictions which describe the production of b-hadrons followed by their decay into $\psi+X$. For $J / \psi$, agreement is generally good, but the theory predicts slightly harder $p_{T}$ spectra than observed in data. For $\psi(2 s)$, the shapes appear to be in satisfactory agreement but the theory predicts higher yields than in data. Also for non-prompt production, there is no observed dependence on rapidity. The following fractional quantities, for which several uncertainties largely cancel in the ratio (e.g. uncertainties on acceptance, efficiency corrections) were also determined:

- the non-prompt fraction $f_{b}^{\psi}(\psi \equiv J / \psi, \psi(2 s))$ defined as the number of non-prompt $\psi$ divided by the total number of produced $\psi$ after weighting corrections:

$$
f_{b}^{\psi}=\frac{p p \rightarrow b+X \rightarrow \psi+X^{\prime}}{p p \stackrel{\text { Inclusive }}{\longrightarrow} b+X \rightarrow \psi+X^{\prime}}=\frac{N_{\psi}^{n p}}{N_{\psi}^{n p}+N_{\psi}^{p}} .
$$

These fractions are shown in figure 5 as a function of the $p_{T}$, in various rapidity intervals, for: (a) $J / \psi$, (b) $\psi(2 s)$. In each rapidity slice, the non-prompt fraction is seen to increase as a function of $p_{T}$ and has no strong dependence on either rapidity or centre-of-mass energy;

- the ratios of $\psi(2 s)$ to $J / \psi$ for prompt and non-prompt production, defined as:

$$
R^{p}=\frac{N_{\psi(2 s)}^{p}}{N_{\psi}^{p}}, \text { and } R^{n p}=\frac{N_{\psi(2 s)}^{n p}}{N_{\psi}^{n p}} .
$$

These ratios, at $\sqrt{s}=7,8 \mathrm{TeV}$, are shown in figure 6 as a function of the $p_{T}$, in various rapidity intervals, for: (a) prompt production, (b) non-prompt production. The non-prompt ratio is shown to be relatively flat across the considered range of $p_{T}$, for each slice of rapidity. For the prompt ratio, a slight increase with $p_{T}$ is observed, with no strong dependence on rapidity or centre-of-mass energy.

\subsection{Non-prompt $J / \psi$ differential production at $\sqrt{s}=13 \mathrm{TeV}$}

A similar study has been extended to data taken in 2015 at $\sqrt{s}=13 \mathrm{TeV}\left(\mathscr{L}=6.4 \mathrm{fb}^{-1}\right)$ [9]. Due to the limited size of the data sample used, the measurements for $\psi(2 s)$ were not considered, and the analysis was restricted to the $p_{T}$ range $8-40 \mathrm{GeV}$ and only to three rapidity intervals: $|y| \leq 0.75$, $0.75 \leq|y| \leq 1.5$ and $1.5 \leq|y| \leq 2.0$. Figure 7 shows the fit results for the $10 \leq p_{T} \leq 11.0 \mathrm{GeV}$ $|y| \leq 0.75$ intervals in which the prompt an non-prompt contributions are clearly visible. The analysis was limited to the determination of the non-prompt fraction $f_{b}^{J / \psi}$ for which several uncertainties largely cancel out. Residual non-cancellation (e.g. geometric acceptance, reconstruction 

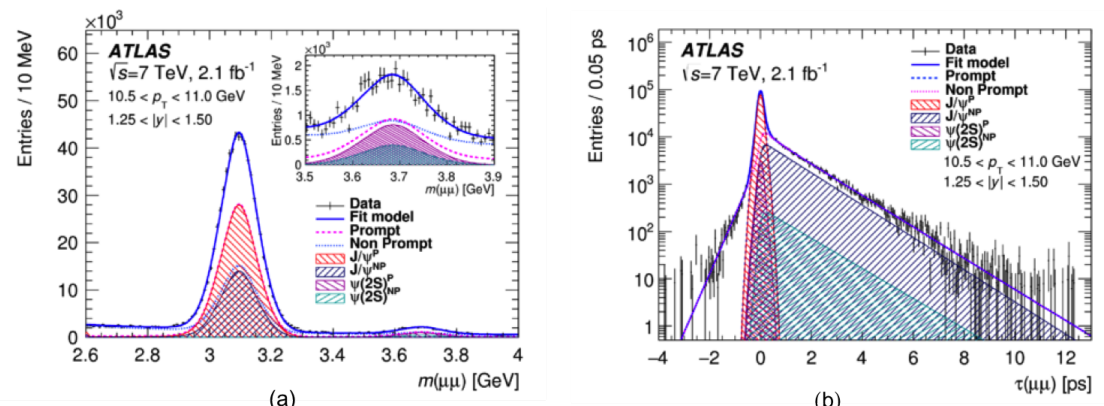

Figure 2: Fit projections [8], for the $10.5 \leq p_{T} \leq 11.0 \mathrm{GeV}-1.25 \leq|y| \leq 1.50$ bin, on: (a) $m_{\mu \mu}$, (b) $\tau_{\mu \mu}$.

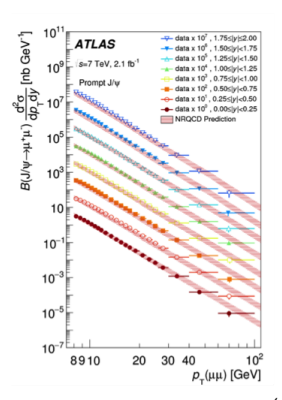

(a)

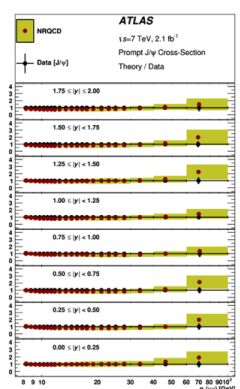

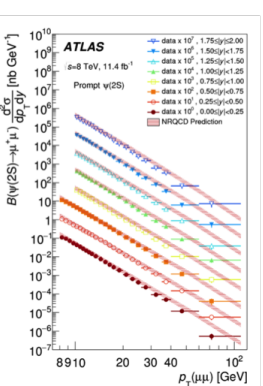

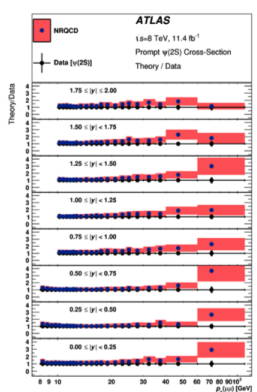

(b)

Figure 3: Example of $p_{T}$-differential cross sections and theory/data ratios in various rapidity interval for prompt production [8]: (a) $J / \psi$ at $\sqrt{s}=7 \mathrm{TeV}$, (b) $\psi(2 s)$ at $\sqrt{s}=8 \mathrm{TeV}$.
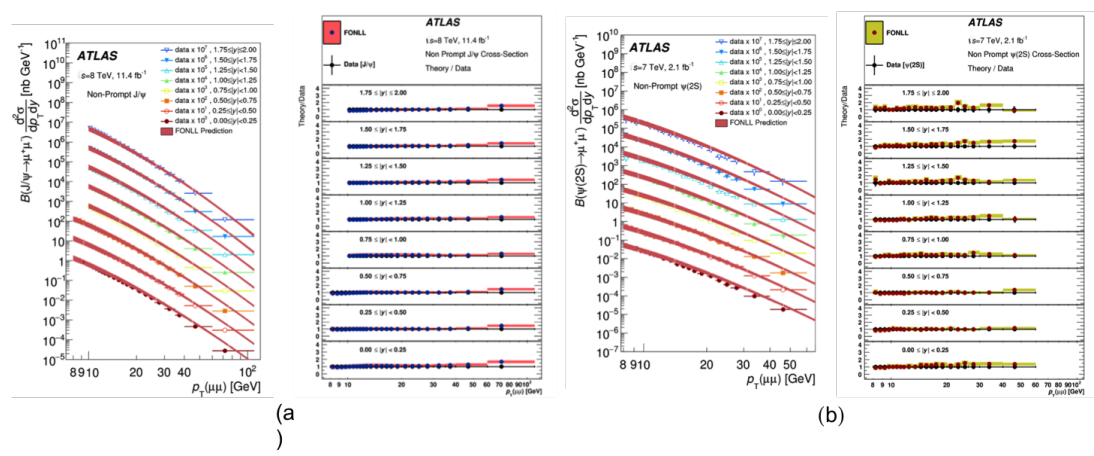

(b)

Figure 4: Example of $p_{T}$-differential cross sections and theory/data ratios, in various rapidity interval for non-prompt production [8]: (a) $J / \psi$ at $\sqrt{s}=8 \mathrm{TeV}$, (b) $\psi(2 s)$ at $\sqrt{s}=7 \mathrm{TeV}$.

efficiencies, etc...) was found to be below $3 \%$ and a systematic uncertainty of $3 \%$ is assigned to the measured non-prompt fractions. The results for $f_{b}^{J / \psi}$ at $\sqrt{s}=13 \mathrm{TeV}$, as function of $p_{T}$ and for the three rapidity intervals under study, are shown in figure 8(a). The non-prompt fraction is found to increase steadily from 0,25 at a $p_{T}=8 \mathrm{GeV}$ to 0.65 at $40 \mathrm{GeV}$, with no significant variation with rapidity observed within the precision of the measurement.

The centre-of-mass energy dependence of this fraction is analyzed comparing these results, for $|y| \leq 0.75$, to previous ATLAS measurement in the same rapidity region at $\sqrt{s}=2.76 \mathrm{TeV}[10]$, the previous measurement at $\sqrt{s}=7 \mathrm{TeV}$ and a CDF measurement [11] in a slightly different rapidity 


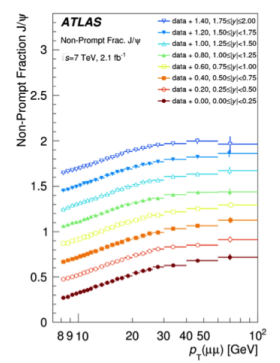

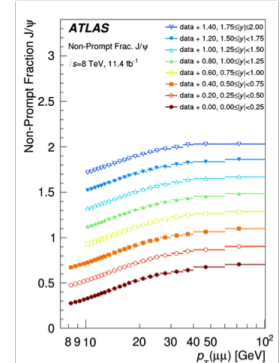

(a)

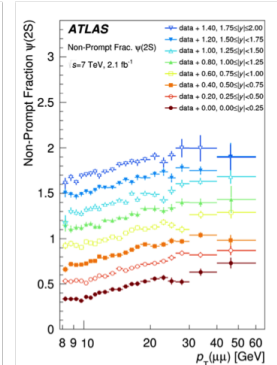

(b)

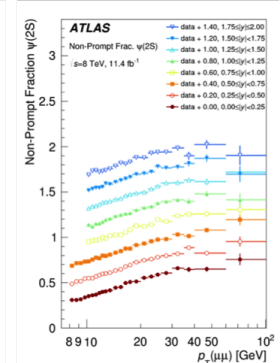

Figure 5: Non-prompt fractions vs $p_{T}$ for various rapidity interval at $\sqrt{s}=7,8 \mathrm{TeV}$ [8]: (a) $J / \psi$, (b) $\psi(2 s)$.
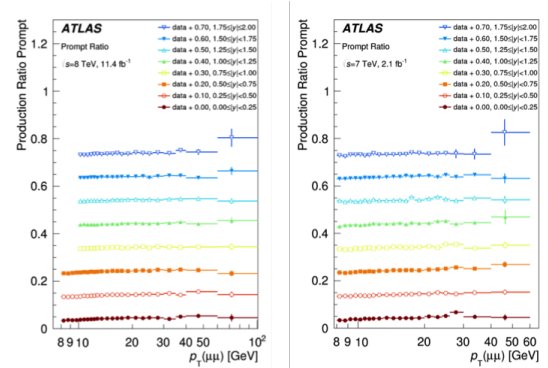

(a)

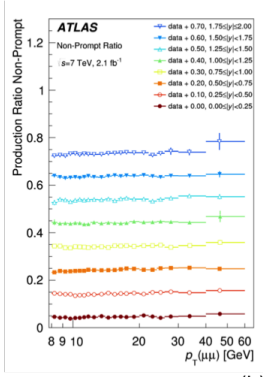

(b)

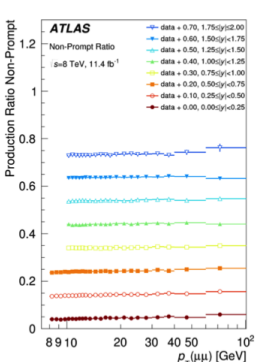

Figure 6: Production ratio $R_{\frac{\psi(2 s)}{J / \psi}}$ vs $p_{T}$ for various rapidity interval at $\sqrt{s}=7,8 \mathrm{TeV}$ [8]: (a) prompt production, (b) non-prompt production.

interval $(|y| \leq 0.60)$ at $\sqrt{s}=1.96 \mathrm{TeV}$. The results are shown in figure $8(\mathrm{~b})$, no significant change in the non-prompt fraction is observed from $\sqrt{s}=7 \mathrm{TeV}$ to $\sqrt{s}=13 \mathrm{TeV}$, while a significant decrease is present between $\sqrt{s}=7 \mathrm{TeV}$ and lower energies.

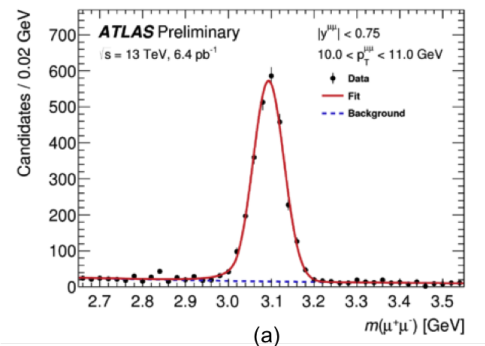

(a)

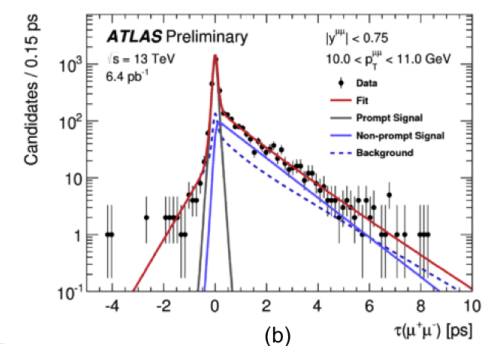

(b)

Figure 7: Fit result [9] for the $10 \leq p_{T} \leq 11 \mathrm{GeV}-|y| \leq 0.75$ interval, projections on: (a) $m_{\mu \mu}$, (b) $\tau_{\mu \mu}$.

\section{Heavy Flavour open state production}

\subsection{Determination of the b-quark fragmentation ratio $\left(\frac{f_{s}}{f_{d}}\right)$ at $\sqrt{s}=7 \mathrm{TeV}$}

The production rate of $B_{s}^{0}\left(B_{d}^{0}\right)$ mesons is a product of the $b \bar{b}$ cross section, the instantaneous luminosity and the probability that the $\bar{b}$-quark is bound to an $s-(d-)$ quark which is denoted as the fragmentation fraction $f_{s}\left(f_{d}\right)$. In a similar fashion, $B^{+}$mesons, $B_{c}$ mesons and $b$-baryons are 


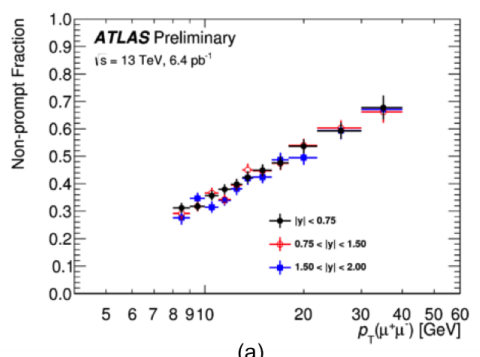

(a)

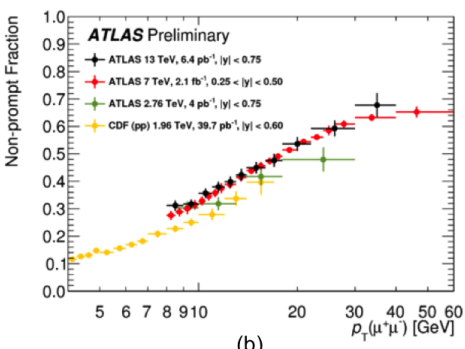

Figure 8: Non-prompt fractions vs $p_{T}$ for $J / \psi$ production [9]: (a) for various rapidity intervals at $\sqrt{s}=$ $13 \mathrm{TeV}$; (b) for various $\sqrt{s}$ in the central rapidity interval.

produced at the LHC with respective fragmentation fractions $f_{u}, f_{c}$ and $f_{\text {baryon }}$. The fragmentation fractions are about $40 \%$ each for $u$ - and $d$-quarks, $10 \%$ for $s$-quarks, at the percent level for $c$-quarks and $\sim 8 \%$ for baryon production, satisfying the constraint $f_{u}+f_{d}+f_{s}+f_{c}+f_{\text {baryon }}=1$. Precise knowledge of the fragmentation fractions is essential for measuring $b$-hadron cross sections and branching fractions at the LHC. In particular for rare decays, such as the branching fraction measurement of $B_{s}^{0} \rightarrow \mu^{+} \mu^{-}$[12], a precise knowledge of $\left(\frac{f_{s}}{f_{d}}\right)$ is important since it improves the sensitivity of searches for new physics processes beyond the Standard Model (SM).

ATLAS has measured [13] the ratio $\left(\frac{f_{s}}{f_{d}}\right)$ using $B_{s}^{0} \rightarrow J / \psi \phi$ and $B_{d}^{0} \rightarrow J / \psi K^{* 0}$ decays, with a data sample corresponding to an integrated luminosity $2.47 \pm 0.04 \mathrm{fb}^{-1}$ of pp collisions at $\sqrt{s}=7 \mathrm{TeV}$. The ratio $\frac{f_{s}}{f_{d}}$ is extracted from the measured $B_{s}^{0} \rightarrow J / \psi \phi$ and $B_{d}^{0} \rightarrow J / \psi K^{* 0}$ signal yields, $N_{B_{s}^{0}}$ and $N_{B_{d}^{0}}$ which are converted into $B_{s}^{0}$ and $B_{d}^{0}$ meson yields after dividing by the branching fractions of the relevant decays and correcting for the relative efficiency $\mathscr{R}_{e f f}$ that is expressed as a product of acceptance and selection efficiency ratios for the two modes and is determined from Monte Carlo (MC) simulations:

$$
\frac{f_{s}}{f_{d}}=\frac{N_{B_{s}^{0}}}{N_{B_{d}^{0}}} \frac{\mathscr{B}\left(B_{d}^{0} \rightarrow J / \psi K^{* 0}\right)}{\mathscr{B}\left(B_{s}^{0} \rightarrow J / \psi \phi\right)} \frac{\mathscr{B}\left(K^{* 0} \rightarrow K^{+} \pi^{-}\right)}{\mathscr{B}\left(\phi \rightarrow K^{+} K^{-}\right)} \mathscr{R}_{e f f},
$$

where the $J / \psi, \phi$ and $K^{* 0}$ are reconstructed in their $J / \psi \rightarrow \mu^{+} \mu^{-}, \phi \rightarrow K^{+} K^{-}$and $K^{* 0} \rightarrow K^{-} \pi^{+}$ final states ${ }^{4}$, respectively. The invariant mass spectra of the selected candidates are shown in figure 9(a), and the corresponding signal yields are obtained with a maximum likelihood fit to the distributions: $N_{B_{s}^{0}}=6640 \pm 100($ stat. $) \pm 220$ (syst. $)$ and $N_{B_{d}^{0}}=36290 \pm 320($ stat. $) \pm 650$ (syst. $)$. From the world average values [14] of $\mathscr{B}\left(\phi \rightarrow K^{+} K^{-}\right)=0.489 \pm 0.005$ and $\mathscr{B}\left(K^{* 0} \rightarrow K^{+} \pi^{-}\right)=$ $0.66503 \pm 0.00014$, and the efficiency ratio $\mathscr{R}_{\text {eff }}=0.799 \pm 0.010$ we obtain:

$$
\left.\frac{f_{s}}{f_{d}} \frac{\mathscr{B}\left(B_{s}^{0} \rightarrow J / \psi \phi\right)}{\mathscr{B}\left(B_{d}^{0} \rightarrow J / \psi K^{* 0}\right)}=0.199 \pm 0.004(\text { stat. }) \pm 0.008 \text { (syst. }\right),
$$

and the details of the systematic uncertainties are described in [13]. Using the perturbative QCD prediction [16] $\frac{\mathscr{B}\left(B_{s}^{0} \rightarrow J / \psi \phi\right)}{\mathscr{B}\left(B_{d}^{0} \rightarrow J / \psi K^{* 0}\right)}=0.83 \pm 0.03$, the ratio of fragmentation functions is obtained:

$$
\frac{f_{s}}{f_{d}}=0.240 \pm 0.004(\text { stat. }) \pm 0.010(\text { syst. }) \pm 0.017(\text { th }) .
$$

\footnotetext{
${ }^{4}$ Charge conjugation is implied unless stated otherwise
} 
Figure 9(b) (left) shows the ATLAS measurement in comparison with results from LEP, CDF and LHCB [15], their consistency confirms the universality of this quantity. At the present level of accuracy, no dependence on $p_{T}$ of the $B$-meson is observed as shown in figure 9(b) (right).
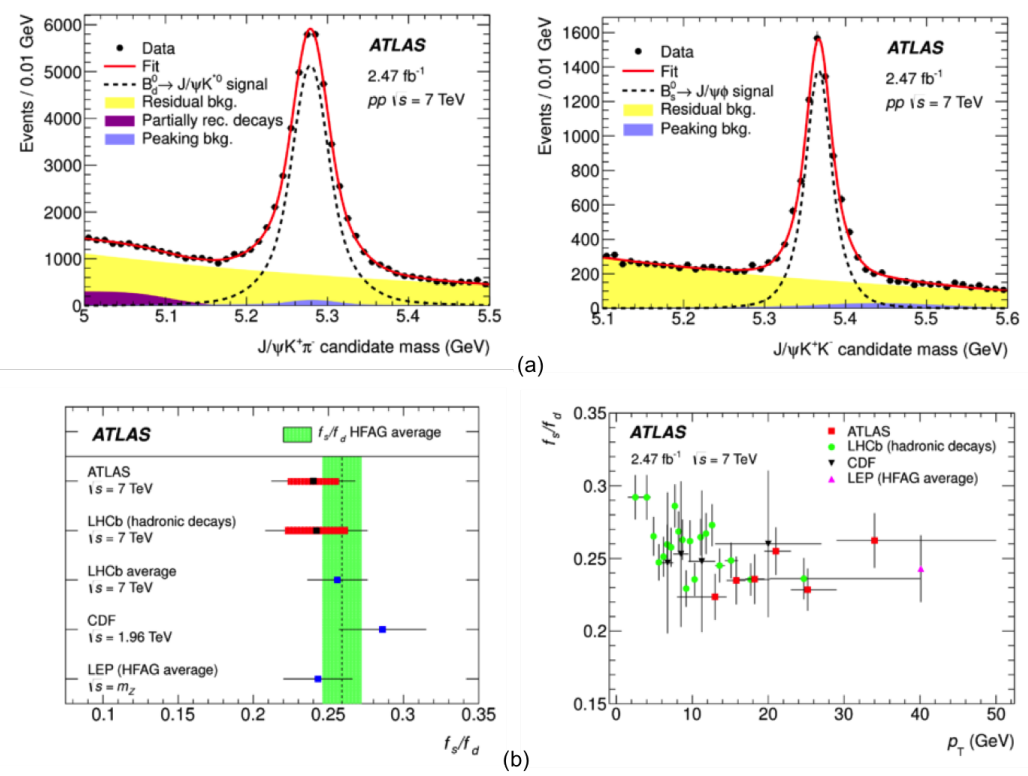

Figure 9: Fragmentation ratio $\left(\frac{f_{s}}{f_{d}}\right)$ [13]: (a) mass spectra for $B_{d}^{0} \rightarrow J / \psi K^{* 0}$ and $B_{s}^{0} \rightarrow J / \psi \phi$; (b) $\frac{f_{s}}{f_{d}}$ determinations and $p_{T}$ dependence.

\section{2 $B^{+}$mass reconstruction in $B^{+} \rightarrow J / \psi K^{+}$decay at $\sqrt{s}=13 \mathrm{TeV}$}

The performance of the ATLAS detector in reconstructing $B$ candidates at $\sqrt{s}=13 \mathrm{TeV}$ has been tested performing a measurement of the $B^{ \pm}$invariant mass through the decay $B^{ \pm} \rightarrow J / \psi\left(\mu^{+} \mu^{-}\right) K^{ \pm}$ [17]. The data sample used in this analysis corresponds to an integrated luminosity of $3.2 \mathrm{fb}^{-1} . B^{ \pm}$ candidates are formed adding a track (kaon candidate), with $p_{T} \geq 3 \mathrm{GeV}$ and $|y| \leq 2.5$, to a previously reconstructed $J / \psi(\mu \mu)$. After quality cut vertexing, $677652 B^{ \pm}$candidates are selected in the mass range $(5.0-5.8) \mathrm{GeV}$, and their mass distribution is shown in figure 10(a). To extract the mass value, a dedicated maximum likelihood fit was performed including the signal and several sources of background. As the mass resolution varies significantly with the rapidity, the mass fit is performed in $16 B^{ \pm}$rapidity intervals $(14 \times \Delta y=0.25$ for $-1.75 \leq|y| \leq+1.75$ and $2 \times \Delta y=0.75$ for $1.75 \leq|y| \leq 2.50$ ). The results are shown in figure $10(\mathrm{~b})$ together with the averaged value. The combined fitted functions are superimposed on figure 10(a). The analysis is repeated with a further quality cut on the transverse length of the candidate $L_{x y} \geq 0.2 \mathrm{~mm}$, giving consistent results. The main systematic uncertainties arise from the modeling of the background in the fit and the choice of the mass window [17]. Finally, the measured value is $m_{B^{ \pm}}=$ $5279.31 \pm 0.11$ (stat.) \pm 0.25 (syst.) $\mathrm{MeV}$, in good agreement with the world average [14], which validates the momentum calibration of the ATLAS tracking with 2015 data.

\section{3 $D^{* \pm}, D^{ \pm}$and $D_{s}^{ \pm}$production cross sections at $\sqrt{s}=7 \mathrm{TeV}$}

The production of $D^{* \pm}, D^{ \pm}$and $D_{s}^{ \pm}$charmed mesons has been measured with the ATLAS de- 

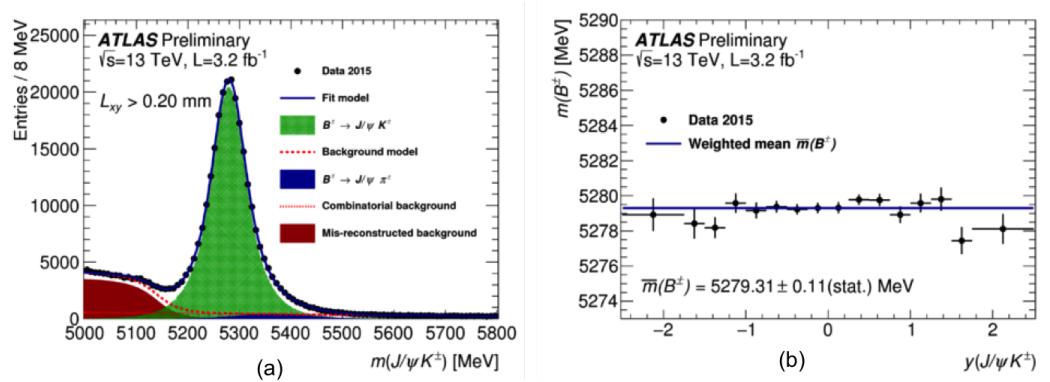

Figure 10: Determination of the $B^{ \pm}$mass [17]: (a) mass distributions for $B^{ \pm} \rightarrow J / \psi K^{ \pm}$; (b) results for different rapidity intervals.

tector in pp collisions at $\sqrt{s}=7 \mathrm{TeV}$ at the LHC [18], using data corresponding to an integrated luminosity of $281 \mathrm{nb}^{-1}$. The $D^{* \pm}, D^{ \pm}$and $D_{s}^{ \pm}$charmed mesons are reconstructed in the range of transverse momentum $3.5 \leq p_{T} \leq 100 \mathrm{GeV}$ and pseudorapidity $\left|\eta_{D}\right| \leq 2.1$. The low $p_{T}$ sample $(\leq 20 \mathrm{GeV})$ is obtained with minimum bias and random triggers, while the high $p_{T}$ sample with $p_{T} \geq 20,30,40 \mathrm{GeV}$ is obtained with a Jet energy trigger with threshold $\geq 5,10,15 \mathrm{GeV}$. No significant differences between results for positively and negatively charged states is observed, all results are presented for the combined samples. The charmed mesons are reconstructed through the exclusive decay modes: (a) $D^{*+} \rightarrow D^{0} \pi_{s}^{+} \rightarrow\left(K^{-} \pi^{+}\right) \pi_{s}^{+}$; (b) $D^{+} \rightarrow K^{-} \pi^{+} \pi^{+}$; (c) $D_{s}^{+} \rightarrow K^{-} K^{+} \pi_{s}^{+}$. The invariant mass distributions for the candidates in both low and high $p_{T}$ regions are shown respectively in figure 11(a)-(c). The yields are obtained with a fit to the mass distribution with a modified Gaussian parametrization for the signal and with threshold or quadratic exponential functions for the background ${ }^{5}$. After correcting for acceptance/reconstruction with simulation, the visible cross sections for a given charmed meson are then extracted in the low- $p_{T}$ range $\left(3.5 \leq p_{T} \leq 20 \mathrm{GeV}\right)$ and high- $p_{T}$ range $\left(20 \leq p_{T} \leq 100 \mathrm{GeV}\right)$. The systematic uncertainty is dominated by the one on the track reconstruction and selection $(\sim 7 \%)$ due to the uncertainty in the detector material description. The results are reported in figure 12 with the predictions of various theoretical models. The FONLL [3], MC@ NLO [19] and POWHEG [20] predictions are consistent with the data within the large theoretical uncertainties, with the central value of the predictions lying below the measurements. The GM-VFNS [4] predictions agree with the data. For $D^{* \pm}$ and $D^{ \pm}$the differential cross sections as a function of $p_{T}$ and $\eta$ are shown in figure 13, the predictions for several models are superimposed. The FONLL, MC@ NLO and POWHEG predictions are generally below the data. They are consistent with the data in the measured $p_{T}$ and $\eta$ ranges within the large theoretical uncertainties. The FONLL and POWHEG predictions reproduce shapes of the data distributions. The $p_{T}$ shape of the MC@NLO prediction is harder than that for the data. The $\eta$ shape of the MC@NLO prediction in the high- $p_{T}$ range differs from the data and all other predictions. The GM-VFNS predictions agree with data in both shape and normalization.

\section{Conclusions}

ATLAS performed several precision measurements on the HF production at LHC to test various

\footnotetext{
${ }^{5}$ Gauss $^{\text {mod }} \propto e^{-0.5 \cdot x^{1+\frac{1}{1+0.5 x}}}$, Threshold $\propto A \cdot x^{B} \cdot \exp \left(B \cdot x+C \cdot x^{2}\right)$, Quadratic $\propto A \cdot \exp \left(B \cdot x+C \cdot x^{2}\right)$
} 

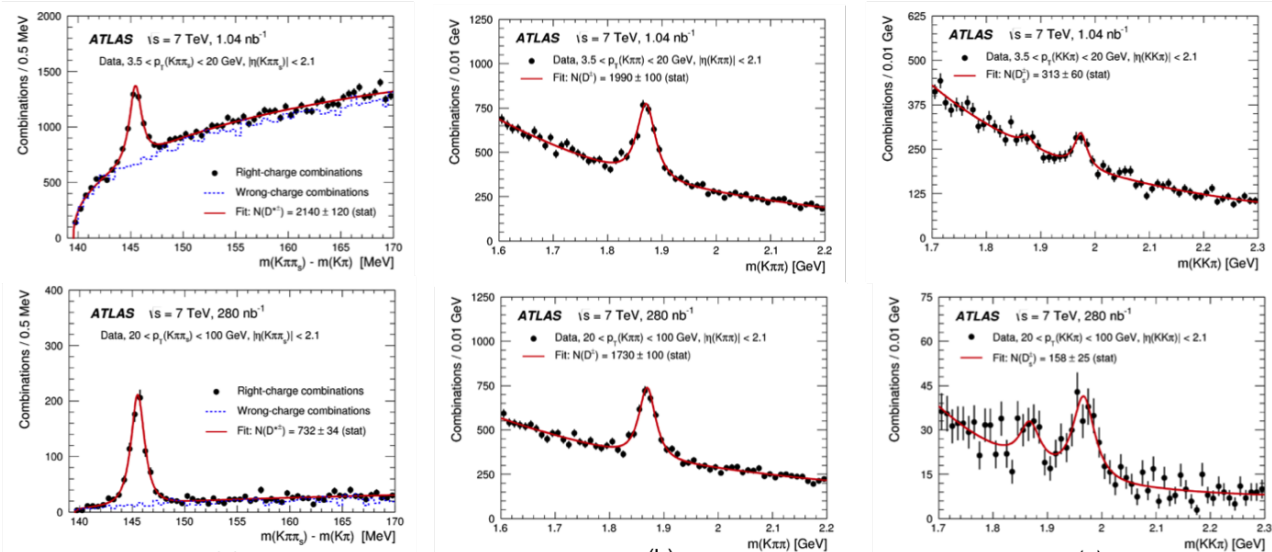

(a)

(b)

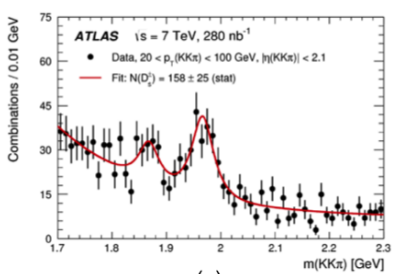

(c)

Figure 11: Invariant mass distributions [18] at $\sqrt{s}=7 \mathrm{TeV}$ in low/high $p_{T}$ regions for: (a) $D^{*+} \rightarrow D^{0} \pi^{+}$, (b) $D^{+} \rightarrow K^{-} \pi^{+} \pi^{+}$, (c) $D_{s}^{+} \rightarrow K^{-} K^{+} \pi^{+}$.

\begin{tabular}{c|c|c|c|c|c|c}
\hline & \multicolumn{2}{|c|}{$\sigma^{\text {vis }}\left(D^{* \pm}\right)$} & \multicolumn{2}{c|}{$\sigma^{\text {vis }}\left(D^{ \pm}\right)$} & \multicolumn{2}{c}{$\sigma^{\text {vis }}\left(D_{s}^{* \pm}\right)$} \\
\hline $\begin{array}{c}\text { Range } \\
\text { [units] }\end{array}$ & $\begin{array}{c}\text { low- } p_{\mathrm{T}} \\
{[\mu \mathrm{b}]}\end{array}$ & $\begin{array}{c}\text { high- } p_{\mathrm{T}} \\
{[\mathrm{nb}]}\end{array}$ & $\begin{array}{c}\text { low- } p_{\mathrm{T}} \\
{[\mu \mathrm{b}]}\end{array}$ & $\begin{array}{c}\text { high- } p_{\mathrm{T}} \\
{[\mathrm{nb}]}\end{array}$ & $\begin{array}{c}\text { low- } p_{\mathrm{T}} \\
{[\mu \mathrm{b}]}\end{array}$ & $\begin{array}{c}\text { high- } p_{\mathrm{T}} \\
{[\mathrm{nb}]}\end{array}$ \\
\hline ATLAS & $331 \pm 36$ & $988 \pm 100$ & $328 \pm 34$ & $888 \pm 97$ & $160 \pm 37$ & $512 \pm 104$ \\
\hline GM-VFNS & $340_{-150}^{+130}$ & $1000_{-150}^{+120}$ & $350_{-160}^{+150}$ & $980_{-150}^{+120}$ & $147_{-66}^{+54}$ & $470_{-69}^{+56}$ \\
FONLL & $202_{-79}^{+125}$ & $753_{-104}^{+123}$ & $174_{-66}^{+105}$ & $617_{-86}^{+103}$ & - & - \\
POWHEG+PYTHIA & $158_{-85}^{+179}$ & $600_{-180}^{+300}$ & $134_{-70}^{+148}$ & $480_{-130}^{+240}$ & $62_{-31}^{+64}$ & $225_{-69}^{+114}$ \\
POWHEG+HERWIG & $137_{-72}^{+147}$ & $690_{-160}^{+380}$ & $121_{-64}^{+129}$ & $580_{-140}^{+280}$ & $51_{-25}^{+50}$ & $268_{-62}^{+107}$ \\
MC@NLO & $157_{-72}^{+125}$ & $980_{-290}^{+460}$ & $140_{-65}^{+112}$ & $810_{-260}^{+390}$ & $58_{-25}^{+42}$ & $345_{-87}^{+175}$ \\
\hline
\end{tabular}

Figure 12: The visible low- $p_{T}\left(3.5 \leq p_{T} \leq<20 \mathrm{GeV}\right)$, and high- $p_{T}\left(20 \leq p_{T} \leq<100 \mathrm{GeV}\right.$, cross sections of $D^{* \pm} D^{ \pm}$and $D_{s}^{ \pm}$production with $|\eta| \leq 2.1$ [18]. The measurements are compared with the GM-VFNS, FONLL, POWHEG+PYTHIA, POWHEG+HERWIG and MC@NLO predictions.

QCD models. These studies are very important because they refer to new kinematic regions (e.g., high $p_{T}$ up to $\sim 10^{2} \mathrm{GeV}$ ) and to channels less affected by uncertainties (e.g., $\psi(2 s)$ ). ATLAS expects to complete the analysis of run-I data and to fully exploit the run-II to probe new interesting phenomena in HF production.

\section{References}

[1] J. Lansberg, Eur. Phys. J. C61 (2009) 693-703

[2] M. Butenschoen and B. A. Kniehl, Nucl. Phys. proc. Suppl. 222-224 (2012) 151-161

B. Gong et al., Phys. Rev. Lett. 110 (2013) 042002

P. Faccioli et al., Phys. Lett. B376(2014) 98-109

[3] M. Cacciari et al., JHEP 1210 (2012) 137

[4] B. A. Kniehl, G. Kramer, I. Schienben and H. Spiersberg, Phys. Rev. D79 (2009) 094009

[5] S. Frixione and B. R. Webber, JHEP 06 (2002) 029

P. Nason, JHEP 11 (2004) 040

[6] ATLAS Collaboration, JINST 3 (2008) S08003 

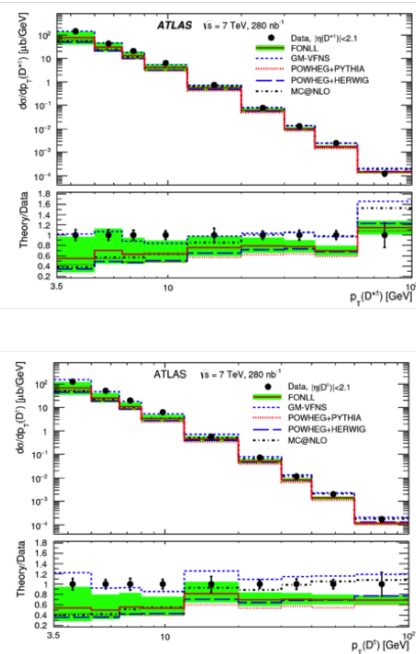

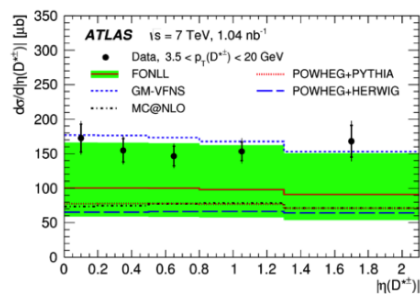

(a)

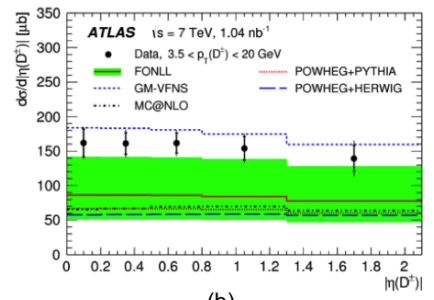

(b)
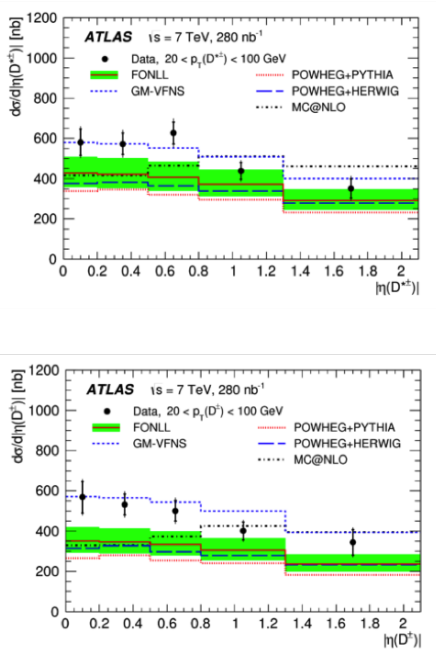

Figure 13: Projections of $p_{T}-\eta$ differential cross sections [18] at $\sqrt{s}=7 \mathrm{TeV}$ for: (a) $D^{*+}$ production, (b) $D^{+}$production.

[7] P. Faccioli et al., Eur. Phys. Jour. C 69 (2010) 657

[8] ATLAS Collaboration, Eur. Phys. J. C (2016) 283

[9] ATLAS Collaboration, ATLAS-CONF-2015-030, http://cds.cern.ch/record/2037967

[10] ATLAS Collaboration, ATLAS-CONF-2015-023, https://atlas.web.cern.ch/Atlas/GROUPS/PHYSICS/CONFNOTES/ATLAS-CONF-2015-023/

[11] CDF Collaboration, Phys. rev. 71 (2005) 032001

[12] ATLAS Collaboration, Phys. Lett. B 713 (2012) 387

R. Aaij et al., LHCb Collaboration, Phys. Rev. Lett. 111 (2013) 101805

CMS Collaboration, Phys. Rev. Lett. 111 (2013) 101804

[13] ATLAS Collaboration, Phys. Rev. Lett. 115 (2016) 262001

[14] K. Olive et al., Review of Particle Physics, Chin. Phys. Rev. C38 (2014) 090001.

[15] Y. Amhis et al., Heavy Flavor Averaging Group, arXiv:1412.7515 [hep-ex]

T. Aaltonen et al., CDF collaboration, public CDF note 10795, 2012

R. Aaij et al., LHCb Collaboration, J. High Energy Phys. 04 (2013) 001

[16] X. Liu, W. Wang and Y. Xie, Phys. Rev. D 89 (2014) 094010

[17] ATLAS Collaboration, ATLAS-CONF-2015-064,http://cds.cern.ch/record/2114830

[18] ATLAS Collaboration, Nucl. Phys. B 907 (2016) 717

[19] G. Corcella et al., JHEP 01 (2001) 010,

S. Frixione, P. Nason and B. R. Webber, JHEP 08 (2003) 007,

S. Frixione, P. Nason and G. Ridolfi, JHEP 09 (2007) 126.

[20] T. Sjostrand, S. Mrenna, P. Skands, JHEP 05 (2006) 026. 